\title{
Nasal high-frequency jet ventilation (NHFJV) as a novel means of respiratory support in extremely low birth weight infants
}

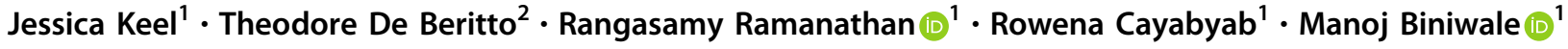

Received: 6 January 2021 / Revised: 26 April 2021 / Accepted: 10 May 2021 / Published online: 14 June 2021

(c) The Author(s), under exclusive licence to Springer Nature America, Inc. 2021

\begin{abstract}
Objective Describe our experience of successfully using nasal high-frequency jet ventilation (NHFJV) in extremely low birth weight infants with respiratory failure.

Study design A retrospective review was conducted on 16 infants with birth weights $<1000 \mathrm{~g}$ who received NHFJV from 2015 to 2019. Successful use was defined as avoiding intubation for at least 72 hours and demonstrating tolerance after being placed on NHFJV.

Results Median gestational age was 24.5 weeks (IQR 24, 25), and weight at the start of NHFJV 1090 g (IQR 905, 1250). NHFJV was used successfully in $13 / 16(81 \%)$ infants with a median duration of 7 days (IQR 3, 12). Days on invasive (30 vs. 186) and noninvasive (46 vs. 81) ventilation were shorter when compared to those who failed the use of NHFJV.

Conclusion This is the first reported case series for the successful use of NHFJV. Our study highlights the feasibility of a potential new mode of noninvasive respiratory support.
\end{abstract}

\section{Introduction}

The use of nasal intermittent positive pressure ventilation (NIPPV) and high-frequency jet ventilation (HFJV) are well-established modes of respiratory support in newborn infants with respiratory failure in the neonatal intensive care unit (NICU). HFJV is a modality that has been typically utilized as a rescue mode in intubated infants not responding to conventional mechanical ventilation to improve clinical comorbidities, particularly pulmonary interstitial emphysema (PIE) and bronchopulmonary dysplasia (BPD) [1]. This modality of ventilation allows for the attainment of higher mean airway pressure, but with minimal volumetric variation in the alveoli allowing for a decrease in supplemental oxygen and resolution of PIE and air leaks [2]. Systematic reviews and meta-analyses have shown that

Manoj Biniwale

biniwale@usc.edu

1 Division of Neonatology, Department of Pediatrics, LAC+USC Medical Center, Keck School of Medicine, University of Southern California, Los Angeles, CA, USA

2 Division of Neonatology, Department of Pediatrics, Mattel Children's Hospital, David Geffen School of Medicine at UCLA, Los Angeles, CA, USA
NIPPV has been shown to be superior to nasal continuous positive airway pressure (NCPAP) to prevent extubation failures [3, 4]. Many studies have demonstrated the improvement in morbidity, mortality, and overall outcomes with the use of both these modalities $[5,6]$.

Nasal high-frequency ventilation (NHFV) is thought to combine the benefits of high frequency ventilation (HFV) and NCPAP. Extremely fast oscillations of very small tidal volumes (less than the physiologic dead space) support the ventilation and provide effective carbon dioxide $\left(\mathrm{CO}_{2}\right)$ removal [7]. This will spare the lung and airways from shear stress and allow the infant to breathe spontaneously without the need for synchronization [7].

While there have been several studies demonstrating the efficacy of nasal high-frequency oscillatory ventilation (NHFOV), the use of nasal high-frequency jet ventilation (NHFJV) has not been reported [7-12]. In this study, we describe our experience with using NHFJV as a rescue mode in patients failing NIPPV or at extubation from invasive mechanical ventilation.

\section{Materials and methods}

A retrospective, observational review was conducted on all inborn, extremely low birth weight (ELBW) infants who 
had received NHFJV. This study included those patients $<1000 \mathrm{~g}$ at birth admitted to the NICU between August 2015 to December 2019. Two centers were included-an academic NICU and an academically affiliated community NICU. Both are level III centers directed by the same group of neonatologists. Data were extracted from the neonatal database (Neonatal Information System, NIS5, Medical Data Systems, Rosemont, PA, USA), electronic medical records, and paper chart review. The following demographic data points were collected: birth weight, gestational age, mode of delivery, use of antenatal steroids, surfactant administration, procedure notes, ventilatory settings, clinical course, diagnoses, and treatment. The study was approved by the local Institutional Review Board.

Data obtained were analyzed using univariate and bivariate statistics. Categorical variables were summarized by counts and percentages, while continuous variables were summarized by medians and interquartile ranges when applicable. Associations of clinical characteristics between "success" and "failure" groups were analyzed using Fischer exact and Wilcoxon rank-sum as appropriate with the use of IBM SPSS Statistics for Windows, version 27 (Armonk, NY, USA). A $p$-value $<0.05$ was considered statistically significant.

The Life Pulse high-frequency jet ventilator (Bunnell Inc., Salt Lake City, UT, USA) was used to provide NHFJV, while the Puritan Bennett ${ }^{\mathrm{TM}} 980$ ventilator (Medtronic, Minneapolis, MN, USA) and Avea (Vyaire Medical, Mettawa, IL, USA) ventilators provided conventional ventilation. The RAM nasal oxygen cannula (Neotech, Valencia, CA, USA) was attached to the LifePort endotracheal tube adapter (Bunnell Inc.) to deliver jet breaths noninvasively (refer to Fig. 1 for individual parts, Fig. 2 for set up). Typical settings were nasal jet peak inspiratory pressure

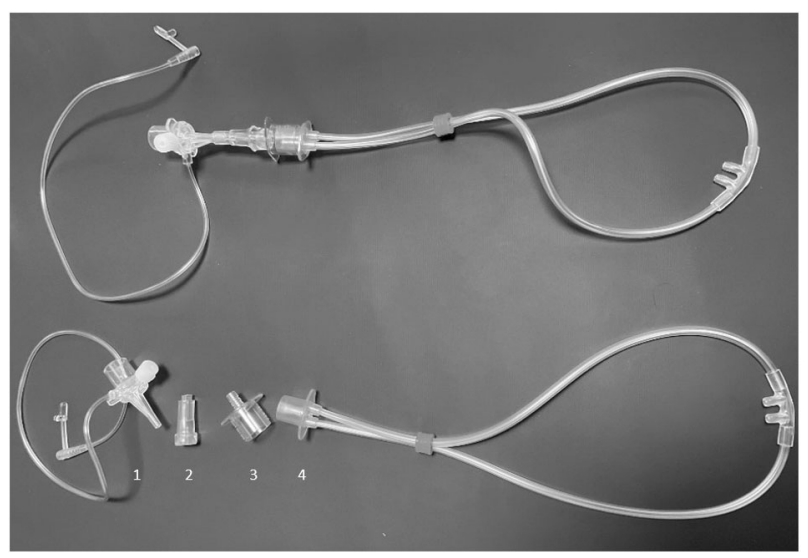

Fig. 1 Set up of tubing apparatus. To deliver jet breaths nasally, a piece of oxygen tubing is cut and affixed to the endotracheal tube adapter, which then attaches to the Neotech RAM nasal cannula. 1 - endotracheal tube adapter; 2 -oxygen tubing; 3-oxygen tubing adapter; 4-RAM nasal cannula.

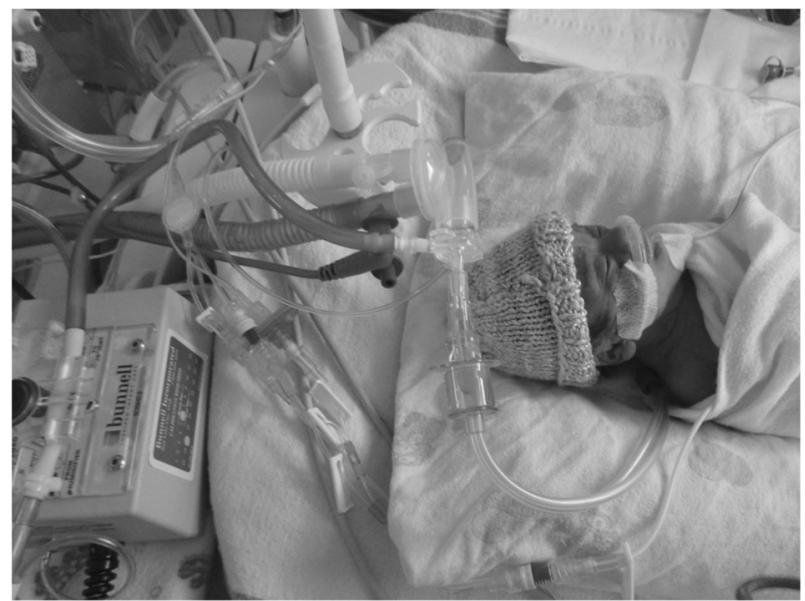

Fig. 2 Infant extubated to nasal high-frequency jet ventilation. Extremely low birth weight infant receiving noninvasive respiratory support via high-frequency jet ventilator (Bunnell Life Pulse ${ }^{\circledR}$ ) in tandem with a conventional ventilator. Written consent to obtain and publish patient photographs was obtained from a legal guardian.

(PIP) of $23-45 \mathrm{cmH}_{2} \mathrm{O}$, jet rate of $240-360$ breaths per minute (bpm), and jet valve on time of 0.03-0.034 s. NIPPV rate was set at $40 \mathrm{bpm}$ with an inspiratory time (IT) of $0.5 \mathrm{~s}$, PIP ranged from 19 to $35 \mathrm{cmH}_{2} \mathrm{O}$, and positive endexpiratory pressure (PEEP) from 8 to $13 \mathrm{cmH}_{2} \mathrm{O}$.

Chest X-rays were obtained after extubation or transition to NHFJV and followed either daily or once every $2-3$ days, depending on the infant's stability. This allowed providers to monitor the degree of lung inflation and address areas of atelectasis. Blood gases were performed at least daily, though some infants did require closer monitoring (every 6-12 h) in the first few days on NHFJV to assess for tolerance and adequate ventilation.

Our primary objective was to assess the feasibility of using NHFJV as an alternative means of noninvasive, escalated respiratory support in ELBW infants. A successful outcome was achieved when the infant remained extubated without worsening hypercarbia or hypoxia for at least 72 hours after being placed on NHFJV. If the infant had not previously been receiving invasive mechanical ventilation and on NCPAP or NIPPV, a successful outcome was defined as avoiding intubation for at least 72 hours since the initiation of NHFJV or being able to transition back to NIPPV as a weaning mode.

\section{Results}

There were 16 ELBW infants (ten males and six females) who received NHFJV. The median birth weight was $686 \mathrm{~g}$ (IQR 508, 746) and the median gestational age was 24.5 weeks (IQR 24, 25). The majority of patients (94\%) were exposed to antenatal steroids and received early rescue 
Table 1 Demographic summary of patients placed on nasal highfrequency jet ventilation.

\begin{tabular}{|c|c|c|c|c|c|c|c|}
\hline Patient number & $\begin{array}{l}\text { Gestation, } \\
\text { weeks/birth } \\
\text { weight, g }\end{array}$ & Gender & $\begin{array}{l}\text { Received } \\
\text { antenatal } \\
\text { steroids }\end{array}$ & $\begin{array}{l}\text { Mode of } \\
\text { Delivery }\end{array}$ & $\begin{array}{l}1 \text { and } 5 \mathrm{~min} \\
\text { Apgar }\end{array}$ & $\begin{array}{l}\text { Small for } \\
\text { gestational age }\end{array}$ & $\begin{array}{l}\text { Surfactant/ } \\
\text { \# of doses }\end{array}$ \\
\hline 1 & $23 / 530$ & Male & Yes & Vaginal & 2,5 & No & Yes/4 \\
\hline 2 & $23 / 480$ & Female & Yes & Vaginal & 4,6 & Yes & Yes/3 \\
\hline 3 & $25 / 630$ & Male & Yes & Cesarean & 4,7 & No & Yes/2 \\
\hline 4 & $25 / 750$ & Male & Yes & Cesarean & 3,6 & No & Yes/3 \\
\hline 5 & $26 / 500$ & Male & Yes & Cesarean & 3,7 & Yes & Yes/1 \\
\hline 6 & $24 / 730$ & Male & Yes & Cesarean & 5,6 & No & Yes/1 \\
\hline 7 & $24 / 430$ & Female & Yes & Cesarean & 2,3 & Yes & Yes/2 \\
\hline 8 & $27 / 910$ & Male & Yes & Cesarean & 5,8 & No & Yes/3 \\
\hline 9 & $23 / 565$ & Male & Yes & Vaginal & 3,6 & No & Yes/2 \\
\hline 10 & $25 / 696$ & Female & Yes & Vaginal & 2,6 & No & Yes/2 \\
\hline 11 & $25 / 920$ & Female & No & Cesarean & 3,5 & No & Yes/3 \\
\hline 12 & $24 / 755$ & Female & Yes & Cesarean & 1,4 & No & Yes/2 \\
\hline 13 & $24 / 708$ & Male & Yes & Vaginal & 2,5 & No & Yes/2 \\
\hline 14 & $26 / 675$ & Male & Yes & Vaginal & 6,8 & Yes & No \\
\hline 15 & $24 / 405$ & Male & Yes & Cesarean & 2,5 & Yes & Yes/4 \\
\hline 16 & $25 / 735$ & Female & Yes & Vaginal & 2,4 & No & Yes/3 \\
\hline
\end{tabular}

surfactant (94\%) with a median number of 2 doses. Baseline patient characteristics are shown in Table 1.

\section{Indications for NHFJV}

Infants were placed on NHFJV for two reasons: first, as a primary prophylactic mode of ventilation after extubation from invasive high-frequency jet ventilation, or second, as a rescue mode to avoid endotracheal intubation when the primary mode of noninvasive respiratory support was deemed inadequate despite maximal settings. In the rescue mode group, patients 8 and 14 (Table 2) never had an endotracheal tube (aside from surfactant administrations for patient 8) and successfully avoided intubation throughout the duration of their NICU stay with the use of NHFJV.

The median day of postnatal age when NHFJV initiated was 30 days (IQR 23, 43) and the median weight at the start of NHFJV was $1090 \mathrm{~g}$ (IQR 905, 1250). There were 9 (56\%) patients extubated directly to NHFJV. Patients 1 and 9 (Table 2) had failed a prior extubation attempt to NIPPV. Two infants with unplanned extubations from invasive HFJV were placed on NHFJV rather than undergoing reintubation.

Seven $(44 \%)$ patients received NHFJV as a rescue mode after maximizing prior settings for a variety of reasons including: worsening hypercarbia (most common), increased work of breathing, increased frequency of apnea, bradycardia, and oxygen desaturation events requiring vigorous stimulation, a higher fraction of inspired oxygen $\left(\mathrm{FiO}_{2}\right)$ and worsening atelectasis or PIE on chest X-ray. Six patients had been on NIPPV previously while one (patient 8 ) had been on NHFOV before switching to NHFJV.

\section{Initial NHFJV settings}

Initial settings on NHFJV were higher by $2-10 \mathrm{cmH}_{2} \mathrm{O}$ for PIP and $1-3 \mathrm{cmH}_{2} \mathrm{O}$ for PEEP compared to invasive HFJV settings. The jet valve on time, was set at $0.03 \mathrm{~s}$ for NHFJV while the IT for NIPPV was set at $0.5 \mathrm{~s}$. The backup rate provided by NIPPV was set at $40 \mathrm{bpm}$.

\section{Clinical outcomes}

Nasal HFJV was used successfully in 13 (81\%) infants with a median duration of 7 days (IQR 3, 12). Early discontinuation within a 24 hour period occurred in 3 (19\%) infants secondary to equipment failure or intolerance manifested as worsening oxygenation or $\mathrm{CO}_{2}$ retention. Of the three cases, patients 2 and 5 improved once transitioned to NIPPV, while patient 11 required reintubation (Table 2). In total, only 1/16 (6\%) infants required intubation after being placed on NHFJV.

The successful use of NHFJV was associated with a decreased number of median invasive ventilator days (30 vs 186) and noninvasive ventilator days (46 vs. 81) when compared to those (patients 2, 5, and 11) who failed NHFJV. A comparison summary of "success" versus "failure" groups is provided in Table 3. There were no significant differences between the two groups except for the duration of NHFJV and total noninvasive ventilation days. A diagnosis of PIE was present in $6(38 \%)$ infants. While all infants had a diagnosis of BPD at 36 weeks postmenstrual age, only 5 of $15(33 \%)$ surviving infants required home oxygen upon discharge including one patient (patient 5) who required a tracheostomy. One infant (patient 2) expired during the 


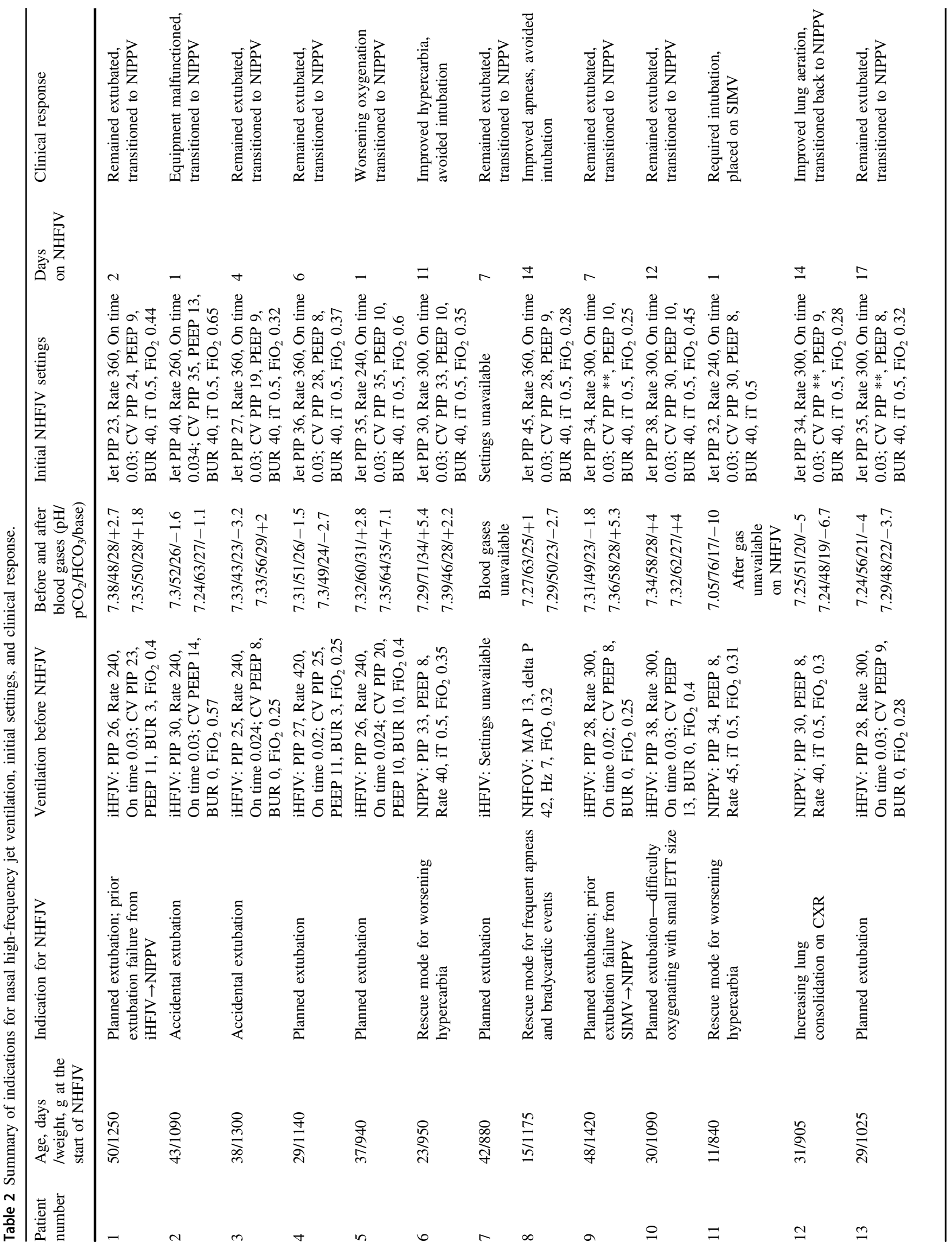




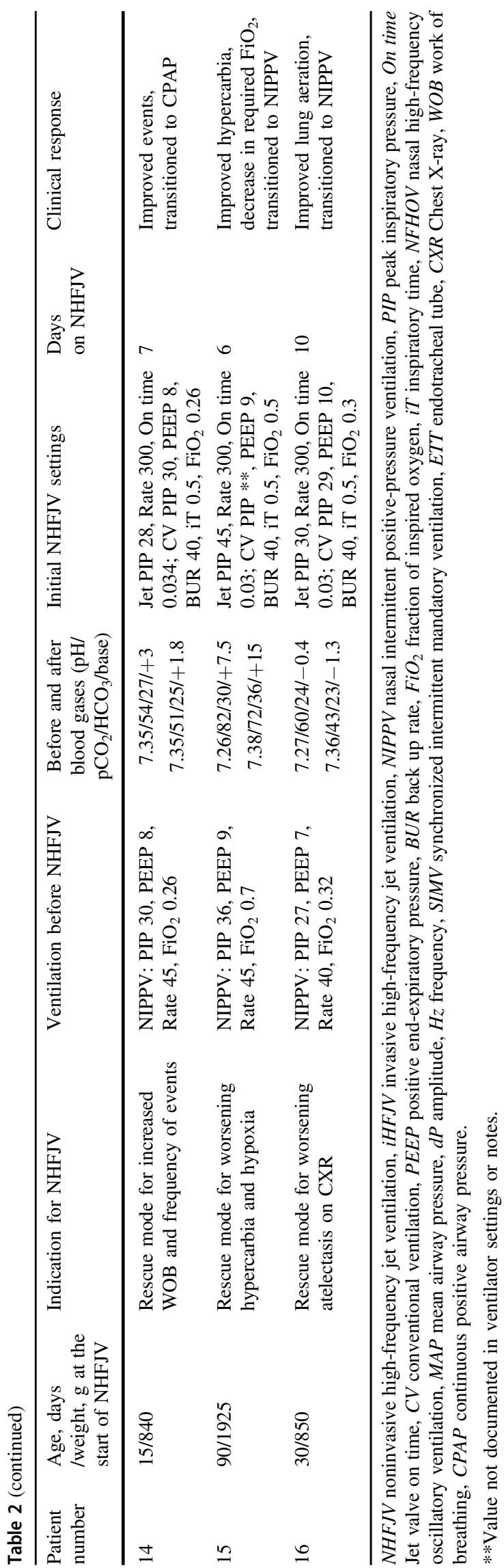

Table 3 Characteristics of patients placed on nasal high-frequency jet ventilation (successful versus failure groups).

\begin{tabular}{|c|c|c|c|}
\hline Groups, $n(\%)$ & Success, 13 (81) & Failure, 3 (19) & $P$-value \\
\hline Birth weight, $\mathrm{g}^{\mathrm{a}}$ & $696(548,743)$ & $500(480,920)$ & 0.80 \\
\hline Gestational age, weeks ${ }^{\mathrm{a}}$ & $24(24,25)$ & $25(23,26)$ & 0.80 \\
\hline $\begin{array}{l}\text { Postnatal age NHFJV } \\
\text { initiated, day }{ }^{\mathrm{a}}\end{array}$ & $30(26,45)$ & $37(11,43)$ & 0.90 \\
\hline $\begin{array}{l}\text { Weight when NHFJV } \\
\text { initiated, } \mathrm{g}^{\mathrm{a}}\end{array}$ & $1090(893,1275)$ & $940(840,1090)$ & 0.30 \\
\hline Male sex, $n(\%)$ & $9(69)$ & $1(33)$ & 0.52 \\
\hline Antenatal steroids, $n(\%)$ & $13(100)$ & $2(67)$ & 0.19 \\
\hline Cesarean section, $n(\%)$ & $7(54)$ & $2(67)$ & 1.00 \\
\hline $\begin{array}{l}\text { Small for gestational } \\
\text { age, } n(\%)\end{array}$ & $3(23)$ & $2(67)$ & 0.21 \\
\hline 1 min Apgar ${ }^{\mathrm{a}}$ & $2(2,5)$ & $3(3,4)$ & 0.44 \\
\hline 5 min Apgar ${ }^{a}$ & $6(5,7)$ & $6(5,7)$ & 0.70 \\
\hline $\begin{array}{l}\text { Surfactant } \\
\text { administration, } n(\%)\end{array}$ & $12(92)$ & $3(100)$ & 1.00 \\
\hline $\begin{array}{l}\text { Number of } \\
\text { (surfactant) doses, } n^{\text {a }}\end{array}$ & $2(2,3)$ & $3(1,3)$ & 0.90 \\
\hline $\begin{array}{l}\text { Duration of NHFJV, } \\
\text { days }^{\mathrm{a}}\end{array}$ & $7(6,12)$ & $1(1,1)$ & $<0.01$ \\
\hline $\begin{array}{l}\text { Duration of invasive } \\
\text { ventilation, days }{ }^{\mathrm{a}}\end{array}$ & $30(20,43)$ & $186(8,283)$ & 0.30 \\
\hline $\begin{array}{l}\text { Duration of noninvasive } \\
\text { ventilation, days }{ }^{\mathrm{a}}\end{array}$ & $46(42,53)$ & $81(49,163)$ & 0.04 \\
\hline
\end{tabular}

${ }^{\mathrm{a}}$ Median (25th, 75th percentile).

NICU course at 9 months from severe hepatic and renal failure resulting in anasarca and coagulopathy. Nine (56\%) had vasopressor-resistant hypotension requiring postnatal steroids and $12(75 \%)$ had a patent ductus arteriosus (PDA) that was medically or surgically treated. One of the three patients who failed NHFJV (patient 11, Table 1) did not receive any antenatal steroids.

\section{Sedation and feeding tolerance on NHFJV}

All infants previously receiving invasive ventilation were on a fentanyl drip for sedation and able to be weaned upon extubation. About half of infants on noninvasive respiratory support were on a fentanyl drip as well, while the other half did not require any form of sedation. Infants appeared comfortable on NHFJV and had no issues weaning sedation as tolerated, most often by $10-15 \%$ every 48 hours. With the exception of one patient on continuous feeds due to hypoglycemia and 3 who were NPO while on dopamine and/or PDA treatment with indomethacin, all others received bolus gavage feeds and tolerated daily advancements on NHFJV. Two infants had mild abdominal distension prompting abdominal X-rays which showed slightly dilated bowel loops; as their physical exams were otherwise reassuring, feeds were continued and they tolerated advancement. 
Patient 8 had excessive bowel gas while on NHFOV which improved once transitioned to NHFJV.

\section{Discussion}

In this study, we report the first successful use of NHFJV in ELBW infants either as a primary mode of ventilation after extubation or as a rescue mode to avoid intubation. Noninvasive methods of ventilation have become increasingly popular over the past decade in an effort to mitigate adverse effects associated with intubations in preterm infants. These comorbidities often include ventilator-induced lung injury that can ultimately lead to severe BPD, subglottic stenosis, air leak syndromes, and tracheal colonization [13]. In addition for those surviving ELBW infants, pulmonary complications leading to long-term diuretic use, need for home oxygen, susceptibility to infections, and repeated hospitalizations are known problems beyond the neonatal period. If intubation cannot be avoided, reducing the duration of invasive ventilation is of significant importance in this high-risk population in an attempt to minimize injury to their premature and developing lungs.

$\mathrm{HFV}$ is a well-known alternative to conventional mechanical ventilation. Anecdotally, NICUs in the United States seem to employ high frequency oscillatory ventilation at higher rates than HFJV given greater familiarity and ease of use with one machine. Despite inconsistent results, some studies have shown the latter to be an effective rescue from conventional ventilation with decreased need for extracorporeal membrane oxygenation, improvement in patients with air leak syndromes, reduced rates of PIE and respiratory distress syndrome, as well as lower incidences of BPD and patients being discharged home on oxygen $[5,14,15]$.

NHFV is not yet a commonly used mode of respiratory support in most NICUs in the United States given the current lack of knowledge regarding long-term outcomes and robust evidence supported by larger trials. Li et al. conducted a meta-analysis of 8 randomized controlled trials involving 463 preterm infants and reported an overall lower risk of intubation and more effective $\mathrm{CO}_{2}$ clearance in the NHFOV group when compared to NIPPV or biphasic CPAP, though half of these trials involved infants with birth weights above $1500 \mathrm{~g}$ [10]. More recently, a randomized controlled trial comparing NHFOV versus NCPAP in 124 preterm infants with respiratory distress syndrome was published [11]. In this trial of 28-to-34-week gestational age infants, NHFOV did not reduce the need for mechanical ventilation during the first 72 hours after birth compared to NCPAP; however, the duration of noninvasive ventilation in the NHFOV group was significantly shorter [11].

The existing literature is limited to case series with examples of other noninvasive HFV types. In 1998, a study from the Netherlands consisting of preterm and term infants with moderate respiratory failure, NHFV therapy using the high-frequency flow interrupter (HFFI, Infant Star HFV, Infrasonics, Inc. San Diego, CA, USA) was shown to decrease the partial pressure of $\mathrm{CO}_{2}\left(\mathrm{pCO}_{2}\right)$ significantly [16]. Colaizy et al. in 2008 were the first in the United States who treated 14 very low birth weight infants on NCPAP, successfully with nasal HFFI, demonstrating an effective decrease in $\mathrm{pCO}_{2}$ [17]. Furthermore, preclinical studies using a newborn lung model in preterm lambs have shown improved ventilation and oxygenation in the nasal HFV group using an HFFI ventilator (Percussionaire Corp., Sandpoint, ID) versus the mechanical ventilation group with better alveolar formation [18]. Our study is the first to report the successful use of NHFJV using the Life Pulse ventilator in tandem with NIPPV in ELBW infants with respiratory failure, thus providing a potential novel addition to noninvasive respiratory support options. NHFJV following extubation in 40 preterm infants has been registered in ClinicalTrials.gov (Identifier: NCT03558737) and this open-label study comparing NHFJV versus NIPPV is expected to be completed in December 2022.

NHFJV could be an effective mode of ventilation in providing efficient gas exchange and alveolar recruitment while continuing to minimize lung injury with low tidal volumes delivered noninvasively at supraphysiologic frequencies. Unlike its invasive counterpart, NHFJV requires the conventional back up ventilator not only to provide PEEP but also to overcome upper airway resistance. Studies involving noninvasive high-frequency oscillatory ventilation in experimental settings have shown variable pressure transmission through upper airways [7]. We chose to use NIPPV to aid spontaneous breathing as a primary mode of ventilation supplemented by jet ventilation, as opposed to CPAP, to overcome upper airway resistance and assist in lung recruitment. Thus in these rescue scenarios, the backup rate was kept higher at $40 \mathrm{bpm}$ to facilitate respiratory unloading and decrease work of breathing with an IT of 0.5 $\mathrm{s}$ as is standard practice with NIPPV use. For the NHFJV component, a longer IT of $0.03 \mathrm{~s}$ was used due to the higher resistance inherent in the nasal ventilation interface which would result in a prolonged time constant.

Many ELBW infants have complicated, protracted clinical courses and this group was no exception given their extreme prematurity. Out of the three infants who failed NHFJV, one could surmise it was due to severity or progression of the disease as patient 2 expired at 9 months and patient 5 went home with a tracheostomy after a 15-month stay in the NICU; it is interesting to note that the only other infant to fail NHFJV was the only one who did not receive antenatal steroids.

Limitations of this study include its retrospective nature and small sample size from two institutions. In addition, 
there were no matched controls. It is possible that optimization of NIPPV would have resulted in a similar effect, though six patients included in the study were rescued from maximal NIPPV settings with $5(83 \%)$ responding successfully to NHFJV. Lastly, the initiation, selection of NHFJV settings, and method of weaning were left to provider judgment, implicating that further studies and a more standardized protocol are necessary. With increasing experience, however, our staff have become more comfortable in using NHFJV as a rescue mode. We acknowledge there are practical difficulties in knowing how to set up connections using the jet ventilator with a nasal cannula interface. This has been addressed at our institutions with more frequent training of respiratory therapy staff on how to set up, use, and adjust settings to optimize gas exchange using this new non-invasive modality.

In conclusion, our study demonstrates the successful use of NHFJV as a rescue mode of ventilation after extubation or when NIPPV fails in ELBW infants. NHFJV has shown promise in optimizing short-term pulmonary outcomes in these infants while decreasing the need for reintubation and the associated comorbidities related to prolonged invasive mechanical ventilation. Though this study has introduced the feasibility and successful outcome of a novel mode of nasal high-frequency jet ventilation support, randomized controlled trials are needed to confirm its safety and efficacy.

Acknowledgements We would like to thank the respiratory care practitioners, neonatal nurse practitioners, and nursing staff for their help during this study.

Author contributions JK conceptualized and designed the study, conducted the acquisition and interpretation of data, and drafted the initial manuscript. TdB conceptualized and designed the study and assisted in data acquisition and interpretation. RR, RC, and MB conceptualized and designed the study and reviewed the interpretation. All authors reviewed and revised the manuscript, approved the final version as submitted, and agree to be accountable for all aspects of the work.

\section{Compliance with ethical standards}

Conflict of interest RR holds a joint patent with Neotech Products, Inc., and receives royalty payments. $\mathrm{MB}$ received a grant from Vyaire Medical for an unrelated clinical trial. The decision to start NHFV was made by the attending neonatologists. All remaining authors declare no conflicts of interest.

Publisher's note Springer Nature remains neutral with regard to jurisdictional claims in published maps and institutional affiliations.

\section{References}

1. Hutchison AA, Bignall S. Non-invasive positive pressure ventilation in the preterm neonate: reducing endotrauma and the incidence of bronchopulmonary dysplasia. Arch Dis Child Fetal Neonatal Ed. 2008;93:F64-F68.

2. Pokora T, Bing D, Mammel M, Boros S. Neonatal high-frequency jet ventilation. Pediatrics. 1983;72:27-32.

3. Ramaswamy VV, Bandyopadhyay T, Nanda D, Bandiya P, More $\mathrm{K}$, Oommen VI, et al. Efficacy of noninvasive respiratory support modes as postextubation respiratory support in preterm neonates: a systematic review and network meta-analysis. Pediatr Pulmonol. 2020;55:2924-39. https://doi.org/10.1002/ppul.25007

4. Lemyre B, Laughon M, Bose C, Davis PG. Early nasal intermittent positive pressure ventilation (NIPPV) versus early nasal continuous positive airway pressure (NCPAP) for preterm infants. Cochrane Datab Syst Rev. 2016. Art. No.: CD005384

5. Keszler M, Modanlou HD, Brudno S, Clark FI, Cohen RS, Ryan RM. et al. Multicenter controlled clinical trial of high-frequency jet ventilation in preterm infants with uncomplicated respiratory distress syndrome. Pediatrics. 1997;100:593-9.

6. Esmaeilnia T, Nayeri F, Taheritafti R, Shariat M, Moghimpour-Bijani F. Comparison of complications and efficacy of NIPPV and nasal CPAP in preterm infants with RDS. Iran J Pediatr. 2016;25:e32352.

7. De Luca D, Dell'Orto V. Non-invasive high-frequency oscillatory ventilation in neonates: review of physiology, biology and clinical data. Arch Dis Child Fetal Neonatal Ed. 2016;101:F565-F570.

8. Chen L, Wang L, Ma J, Feng Z, Li J, Shi Y. Nasal high-frequency oscillatory ventilation in preterm infants with respiratory distress syndrome and ARDS after extubation: a randomized controlled trial. Chest. 2019;155:740-8.

9. Iranpour R, Armanian A, Abedi A, Farajzadegan Z. Nasal highfrequency oscillatory ventilation (nHFOV) versus nasal continuous positive airway pressure (NCPAP) as an initial therapy for respiratory distress syndrome (RDS) in preterm and near-term infants. BMJ Paediatr Open. 2019;3:e000443 https://doi.org/10. 1136/bmjpo-2019-000443

10. Li J, Li X, Huang X, Zhang Z. Noninvasive high-frequency oscillatory ventilation as respiratory support in preterm infants: a metaanalysis of randomized controlled trials. Respir Res. 2019;20:58.

11. Malakian A, Bashirnezhadkhabaz S, Aramesh MR, Dehdashtian M. Noninvasive high-frequency oscillatory ventilation versus nasal continuous positive airway pressure in preterm infants with respiratory distress syndrome: a randomized controlled trial. J Matern Fetal Med. 2020;33:2601-7.

12. Ali Y, Seshia MM, Ali E, Alvaro R. Noninvasive high-frequency oscillatory ventilation: a retrospective chart review. Am J Perinatol. 2020. https://doi.org/10.1055/s-0040-1718738

13. Aly H. Ventilation without tracheal intubation. Pediatrics. 2009; 124:786-9.

14. Clark RH, Yoder BA, Sell MS. Prospective, randomized comparison of high-frequency oscillation and conventional ventilation in candidates for extracorporeal membrane oxygenation. J Pediatr. 1994;124:447-54.

15. Keszler M, Donn SM, Bucciarelli RL, Alverson DC, Hart M, Lunyong V, et al. Multicenter controlled clinical trial comparing highfrequency jet ventilation and conventional mechanical ventilation in newborn infants with pulmonary interstitial emphysema. J Pediatr. 1991;119:85-93.

16. van der Hoeven M, Brouwer E, Blanco CE. Nasal high frequency ventilation in neonates with moderate respiratory insufficiency. Arch Dis Child Fetal Neonatal Ed. 1998;79:F61-F63.

17. Colaizy TT, Younis UM, Bell EF, Klein JM. Nasal high-frequency ventilation for premature infants. Acta Paediatr. 2008;97:1518-22.

18. Rehan VK, Fong J, Lee R, Sakurai R, Wang ZM, Dahl MJ, et al. Mechanism of reduced lung injury by high-frequency nasal ventilation in a preterm lamb model of neonatal chronic lung disease. Pediatr Res. 2011;70:462-6. 